\title{
Role of Multislice CT in Evaluation of Traumatic Hind-Foot Injuries
}

\author{
Medhat M. Refaat, Ahmed E. Shalaan, Esraa F. Abdl-Khalek
}

\begin{abstract}
Department of diagnostic and interventional radiology, Benha faculty of medicine, Banha University, Egypt.

Correspondence to: Esraa F. Abdl-Khalek, Department of diagnostic and interventional radiology, Benha faculty of medicine, Banha University, Egypt.
\end{abstract}

\section{Email:}

esraafawzybenha@gmail.com

Received: 2 August 2021

Accepted: 11 December 2021

\begin{abstract}
Background: Injuries to the foot and ankle are often missed or underestimated during the initial care for poly-traumatized patients. The aim of this study was to assess the role played by the multi-slice CT in hind foot fractures evaluation .Methods: Our study was involve 30 patients suffering of foot trauma with hind foot fractures, Cases were selected randomly from patients referred to the department of Radio-diagnosis with history of trauma and clinically suspicious cases of hind-foot injuries. Results: There mean age of patients was 31 years. and 18 patients $(60 \%)$ were males and 12 patients $(40 \%)$ were female. the two main injury mechanisms were falling from a height (13 patients [43.4\%]) and a traffic accident (17 patients [56.6\%]). and included 7 ankle fractures, 5 talar fractures, and 18 calcaneal fractures. 6 patients (20\%) had multiple fractures. Of which 4 patients $(67.7 \%)$ had ankle and calcaneal fractures, two patients (33.3\%) had calcaneal and talar fractures. Conclusion: In complex intra-articular distal tibial,
\end{abstract} calcaneal and talar fractures, MDCT demonstrates well the degree of articular surface depression, fragment size and position. Data obtained by from volume-rendered imaging may assist the clinician in planning the surgical approach. Computed tomography is valuable in the diagnosis of cases of occult syndesmotic injury, especially when the patient cannot tolerate stress radiographs.

Keywords: multislice; CT; traumatic; hind-foot; injuries

\section{Introduction}

Conventional radiography has had and still diagnostic evaluation of patients with acute has an essential and dominant role in the ankle and foot trauma. In patients with 
complex ankle and foot fractures, however, CT is a commonly used imaging technique after radiography. The prevalence of complex injuries of the ankle and foot seems to be increasing as a result of the increased use of automobile safety devices, such as seat belts and air bags that decrease mortality and protect the trunk but not necessarily the lower extremities (1).

As a result of technical breakthroughs, MDCT is faster and has better temporal, spatial, and contrast resolution than conventional helical CT. Also, 2D reformats (multiplanar reconstructions) and 3D surface renderings are of excellent quality; and because the image processing is fast, these images can be made almost instantly. The purpose of our study was to assess the MDCT findings and the advantages of MDCT compared with radiography in patients referred to a level 1 trauma center for evaluation of acute ankle and foot trauma (2).

The ankle is one of the most commonly injured joints. Although injuries to the lower extremity exert significant influence on long-term outcome after discharge from the acute care facility, they are often missed or underestimated during the initial care for poly-traumatized patients (3).
Although many of these injuries are purely ligamentous, fractures are not infrequent (3). Radiography often underestimates the extent of injury in the extremities. MDCT in the extremities is helpful in fracture detection, characterization, and treatment planning. On axial CT images alone, it can be difficult to interpret the complex anatomy of the ankle and foot (4).

The development of multi-detector CT (MDCT) has transformed CT from a simple, cross-sectional imaging technique to an advanced, three-dimensional (3-D) imaging modality, enabling excellent 3-D displays. Its advantages over its predecessor, singleslice helical CT, are isotropic imaging capability and ease of interpretation (5).

Extremity imaging has benefited tremendously from isotropic data acquisition. MDCT enables multi-planar reconstruction (MPR) images to be created in any plane with the same spatial resolution as the original sections without degradation of image quality. It is no longer necessary to position patients who are already in pain into uncomfortable positions in order to obtain diagnostic images. In imaging of trauma patients, MPR images are always obtained and are at least as important as the axial images (6). 
The 3-D images help in understanding the spatial relations, which is important for fracture classification and for preoperative planning. 3-D imaging is an important adjunct to axial and MPR images for the evaluation of spatial relations. However, 3D images alone should not be used for the detection and characterization of fractures (5).

The aim of this work was to assess the role played by the multi-slice CT in hind foot fractures evaluation.

\section{Patients and methods}

This prospective study, was conducted during the period from February 2020 to February 2021.Our study was done on patients suffering of foot trauma with hind foot fractures. Cases were selected randomly from patients referred to the Department of Radio-diagnosis with history of trauma and clinically suspicious cases of hind-foot injuries.

All the participating cases in the study had written informed consent, and the study was approved by the Ethical committee of Benha Faculty of Medicine.

\section{Every patient was subjected to the following:-}

- Full history talking.
- Clinical examination.

- Plain radiography.

- Multislice CT examination.

\section{Inclusion criteria:}

- Patients proven to have hind-foot fractures.

- All patients referred to the department of Radio-diagnosis with history of trauma and clinically suspicious cases of hind-foot fractures.

- Cases of all age groups.

- Patients approve to be enrolled in this study.

\section{Exclusion criteria:}

- Patient with no hind-foot fractures.

- Any absolute contraindication for radiation, e.g: pregnant women.

- Previously operated calcaneus or talus bones that might have altered the normal anatomy.

- Patients refusing to be enrolled in this study.

\section{Methodology}

\section{Patient preparation:}


- Detailed explanation of the procedure to the patient.

- Conventional radiography as plain radiography.

\section{Technique and CT imaging data acquisition:}

\section{X-ray:}

All patients were assessed with routine plain radiographs, including lateral and axial Harris views of the hind-foot. Additional views were obtained if indicated like oblique and Mortise views

\section{CT scan:}

Scanning the patient was obtained by multidetector CT while the patient lying supine in the scanner or as the patient status could allow. Reconstruction CT planes were obtained and reformatted images in coronal, axial and sagittal views were produced. 3D reformatted images were also frequently produced.

All patients underwent the same MDCT examination protocol using $0.5 \mathrm{~mm}$ collimation scanner with a gantry rotation speed of $400 \mathrm{~ms} /$ rotation, range of box 450 500, image thickness $0.5 \mathrm{~mm}$ and $0.5 \mathrm{~mm}$ spacing at subtalar joint and $1 \mathrm{~mm}$ above and below it, standard pitch factor of 0.641 , reconstruction interval $0.5 \mathrm{~mm}$ and total exposure time 6-8 s. Each scan was obtained with a tube voltage of $120 \mathrm{kV}$ and 250 mAs. Images were carried in both bone window for fracture classification and associated other bony injuries and soft tissue window for tendon entrapment and any soft tissue abnormalities.

The data were reconstructed into $0.75 \mathrm{~mm}$ slice images, resulting in a total of 400-500 slices. The threshold value for voxels was selected by threshold segmentation ( -600 to $1500 \mathrm{HU})$ that was appropriate to differentiate muscles, tendons and bones.

The acquired images were transferred to a separate workstation for post processing (advantage workstation, AW) with manufacturer-provided software that allowed generation of 2D and 3D images: 2D multiplanar reconstruction (MPR) in the sagittal and coronal planes as well as sagittal reformatted images of the calcaneus was prescribed for the axial images at the level of the ankle joint.

Coronal images were reformatted perpendicular to the sagittal images, also in reference to the ankle joint. All images were evaluated under standard window settings for calcaneal fractures (Window level 300 HU, Window Width $1300 \mathrm{HU}) .3 \mathrm{D}$ volume 
rendered images were done as they are essential for 1 - full extent of the fracture, 2 - relationship of the fracture to the articular surfaces and 3 - searching for displaced calcaneal fragments.

\section{Results}

This study was conducted Benha University Hospitals on 30 patients with a hind foot trauma. The mean age of patients was 46 years. Eighteen patients $(60 \%)$ were males and 7 patients $(38.9 \%)$ were female (Table 1).

In our patient population, the two main injury mechanisms were falling from a height (13 patients [43.4\%]) and a traffic accident (17 patients [56.6\%]) (Table 2).

The present study included 7 ankle fractures, 5 talar fractures, and 18 calcaneal fractures (Figure 1).

According to Associated injuries in the present study's sample, 16 patients $(23.3 \%)$ had soft tissue edema, 2 patients $(11.1 \%)$ had muscle injuries and 3 patients (16.7\%) had tendon injuries (Table 3).

Six patients (20\%) had multiple fractures. Of which 4 patients $(66.7 \%)$ had ankle and calcaneal fractures, two patients $(33.3 \%)$ had calcaneal and talar fractures, Intraarticular fracture types, frequency and percentage according to Sanders classification are showed in table 4.

\section{Case 1:}

A 30 year old male patient presented to the emergency department with dislocated left ankle due to falling from height. \# Sagittal multi-detector CT scan of the left ankle (a) showed suluxated tibio-talar articulation (red arrow). \# Coronal multidetector CT scan (b) shows widened medial clear space $(6.1 \mathrm{~mm})$ (yellow arrow). Note the associated intraarticular fracture of the lateral process of the talus (blue arrow). \# Axial multi-detector CT scan (c) showed the associated fracture of the lateral process of the talus (blue arrow). \# VR image of the left ankle (d) shows the subluxated ankle (red arrow). Note, the absence of malleolar fractures. \# VR image of the ankle tendons (e) shows associated peroneal tendon dislocation (green arrow) and demonstrates well the intra-articular extension of the lateral process fracture (blue arrow) (Figure 2)

\section{Case 2:}

Fig. 3. A 35-year old female patient presented after a fall from a height. Her plain $\mathrm{X}$ ray and $\mathrm{CT}$ scan showed an intraarticular fracture (Sanders type IIB) of the right calcaneus. (a) Lateral radiograph of the right foot showed a fracture line of the 
calcaneus reaching the posterior facet and the inferior surface (arrows). (b) Sagittal multidetector CT scan of the right hindfoot revealed a comminuted fracture of the calcaneus extending to the subtalar joint (arrows). (c) Coronal multidetector CT scan of the right hindfoot showed a comminuted calcaneal fracture with a primary fracture line (arrows) oriented at the middle of the posterior facet of the subtalar joint. (d) Axial multidetector CT scan showed a right calcaneal fracture oriented at the middle of the subtalar joint (red arrow). Another fracture line interrupted the lateral surface (blue arrow) (Figure 3)

Table (1) General characteristic

\begin{tabular}{lll}
\hline Age (years) & Mean \pm SD & $46 \pm 8$ \\
Gender & Males $n(\%)$ & $18(60)$ \\
& Females $\mathrm{n}(\%)$ & $12(40)$ \\
\hline Total & & 18 \\
\hline
\end{tabular}

Table (2) Mechanism of injury

\begin{tabular}{lll}
\hline Mechanism of injury & $\begin{array}{l}\text { falling from a height } \\
\text { traffic accident } \mathrm{n}(\%)\end{array}$ & $13(43.4)$ \\
\hline Total & & $17(56.6)$ \\
\hline
\end{tabular}

Table (3) Associated injuries

\begin{tabular}{llc}
\hline Associated injuries & Soft tissue edema n (\%) & $16(23.3)$ \\
& Muscle injuries n (\%) & $2(11.1)$ \\
Total & Tendon injuries n (\%) & $3(16.7)$ \\
\hline
\end{tabular}

Table (4) Intra-articular fracture types, frequency and percentage according to Sanders classification.

\begin{tabular}{|c|c|c|}
\hline \multirow{4}{*}{ Sanders types } & $\begin{array}{l}\text { Type I : Nondisplaced posterior facet (regardless of number of } \\
\text { fracture lines) n }(\%)\end{array}$ & $2(11.1)$ \\
\hline & $\begin{array}{l}\text { Type II : One fracture line in the posterior facet (two fragments) } \\
\mathrm{n}(\%)\end{array}$ & $3(16.7)$ \\
\hline & $\begin{array}{l}\text { Type III Two fracture lines in the posterior facet (three fragments) } \\
\mathrm{n}(\%)\end{array}$ & 7 (38.9) \\
\hline & $\begin{array}{l}\text { Type IV Comminuted fracture in posterior facet (four or more } \\
\text { fragments) } \quad \mathrm{n}(\%)\end{array}$ & $6(33.3)$ \\
\hline Total & & 18 \\
\hline
\end{tabular}




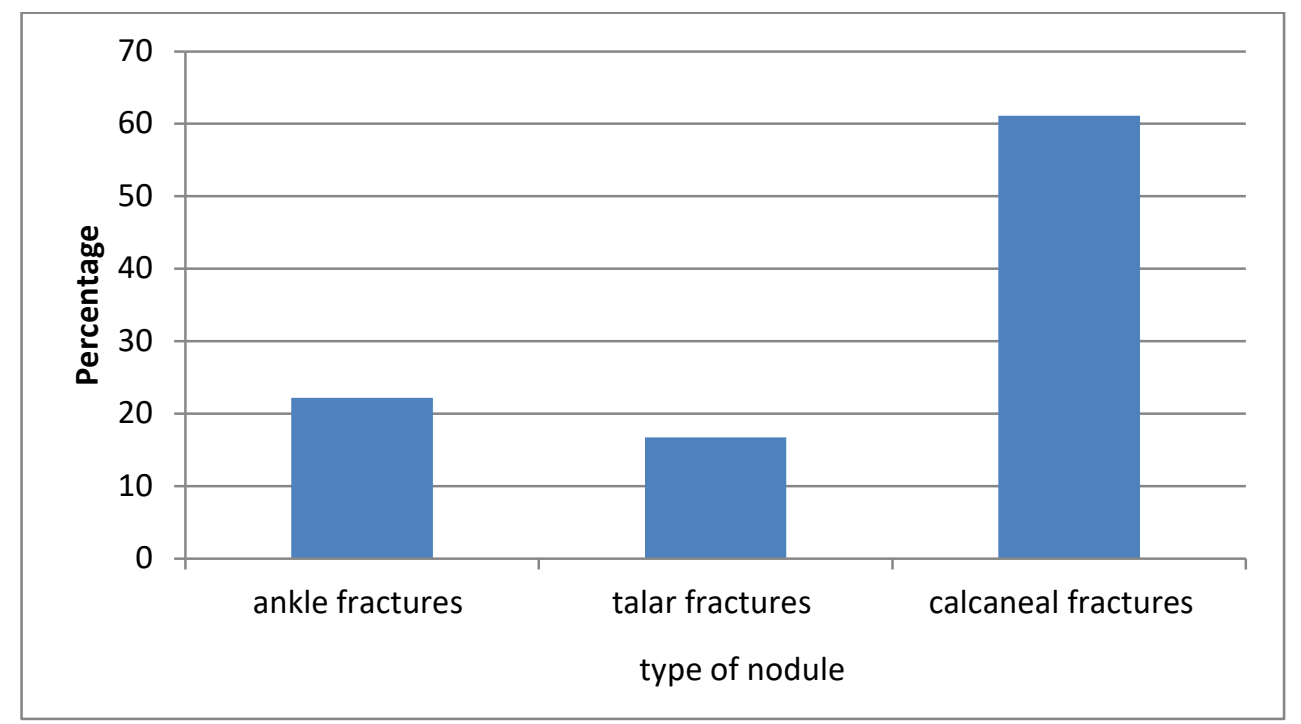

Figure (1) Types of fracture

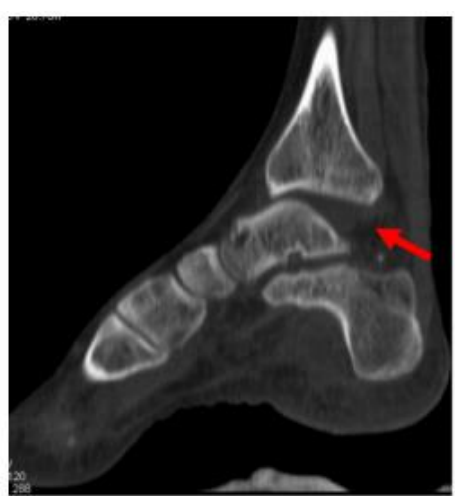

(a)

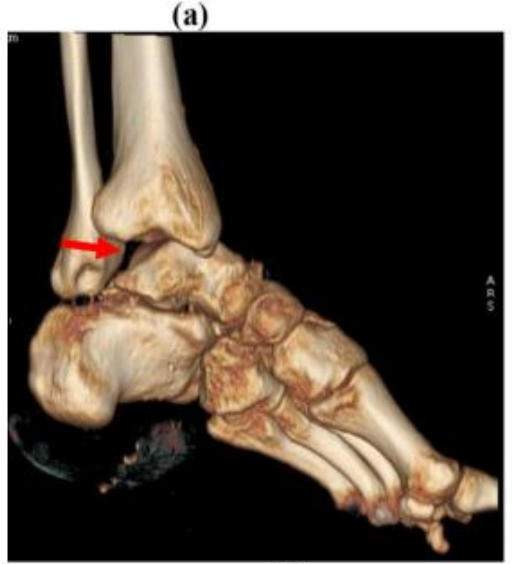

(d)

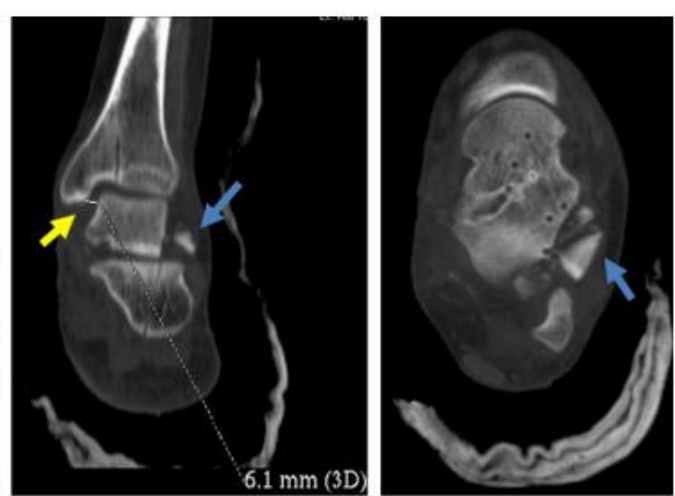

(b)

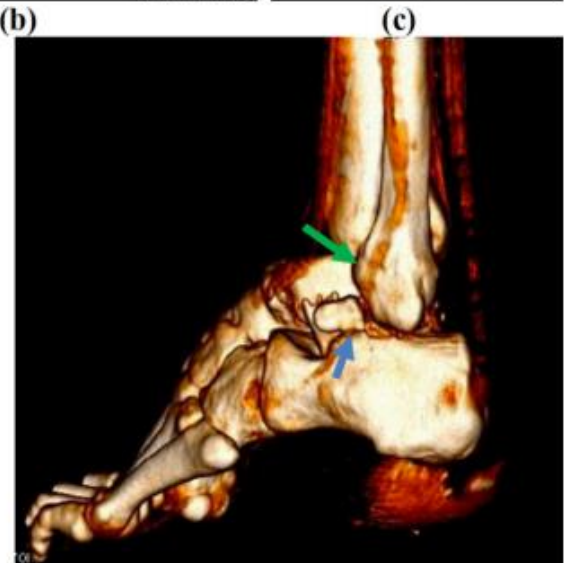

(e)

Fig 2 : case 1 : dislocated left ankle 
Multislice CT in hind-foot injuries, 2022 
(a)

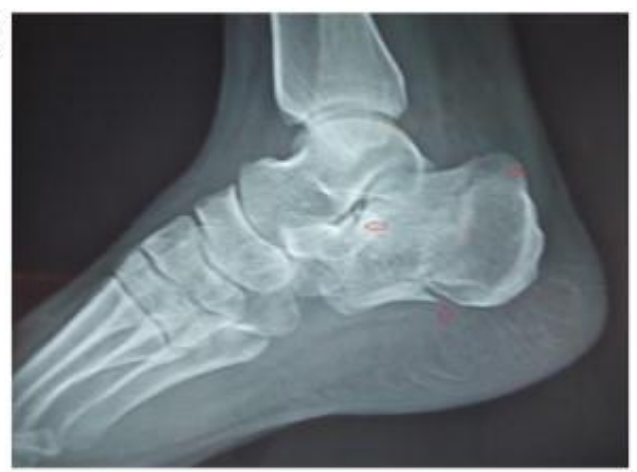

(b)

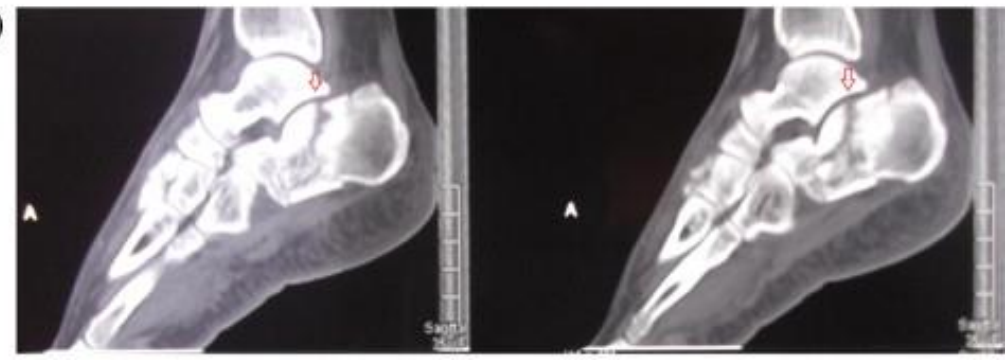

(c)

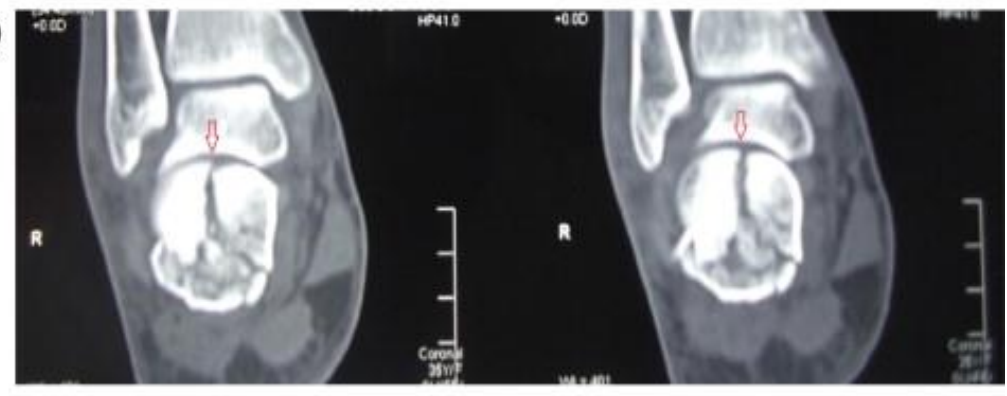

(d)

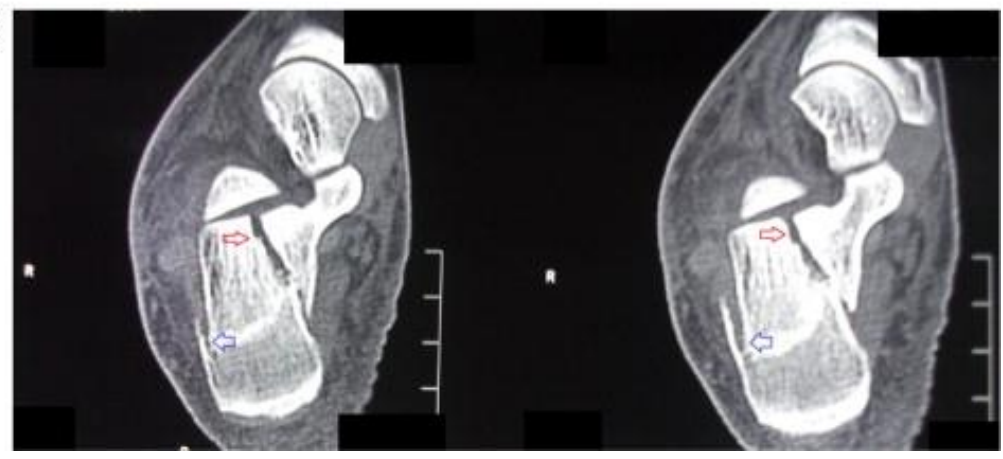

Fig 3: case 2 : Comminuted calcaneal fracture

\section{Discussion}

Multi Detector Computed Tomography (MDCT) images have both higher sensibility and specificity than radiography. CT images can be more easily interpreted even when anatomical relations are subverted. MPR images should be performed along the anatomical axes of the foot. MDCT evaluation with MPR and VRT 
reconstruction are recommended to best assess fracture, anatomical relationship, degree of comminution, eventual intra articular loose bodies. CT is also needed to guide management decisions and for surgical planning (7).

The aim of this work was to assess the role played by the multi-slice CT in hind foot fractures evaluation.

This study was conducted Benha University Hospitals on 30 patients with a hind foot trauma.

There mean age of patients was 31 years. Eighteen patients $(60 \%)$ were males and 12 patients $(40 \%)$ were female. In our patient population, the two main injury mechanisms were falling from a height (13 patients [43.4\%]) and a traffic accident (17 patients [56.6\%]). The present study included 7 ankle fractures, 5 talar fractures, and 18 calcaneal fractures. 6 patients $(20 \%)$ had multiple fractures. Of which 4 patients $(67.7 \%)$ had ankle and calcaneal fractures, two patients $(33.3 \%)$ had calcaneal and talar fractures

Obtaining an internal oblique view in addition to the anteroposterior and lateral projections increases the sensitivity of conventional radiography in the detection of ankle fractures, but in patients with polytrauma and in those with severe comminuted ankle fractures, the appropriate positioning of the ankle is difficult. (8)

On MDCT, the position of the ankle is not crucial because of the high quality of the reformats. The patterns of articular fracture in the distal part of the tibia, especially in comminuted pilon fractures, can be difficult to manage and therefore MDCT scans with multiplanar reconstructions enhance the preoperative assessment of the fracture and operative decision making. The calcaneus is the most commonly fractured foot bone (9).

Calcaneal fractures are the result of highenergy trauma, usually due to a fall from a height or to a motor vehicle accident, which was also seen in the present study.

Nonoperative treatment is best reserved for nondisplaced calcaneal fractures; however, for patients who have displaced intraarticular fracture fragments, nonoperative treatment offers little chance of a return to normal function because a calcaneal mal-union will develop (10).

In intraarticular calcaneal fractures, the degree of the depression of the posterior calcaneal facet may often be underestimated on lateral radiographs and also on coronal CT images (11). 
Multiplanar reconstructions in sagittal and coronal planes show the exact position of the posterior facet and joint malalignment without superimposed structures, thus helping decision making between operative and non-operative treatments The overall sensitivity of conventional radiography in the detection of talar fractures is only moderate, and making the diagnosis of associated intraarticular fractures of the subtalar or talonavicular joint with a subtalar joint dislocation is even more difficult on conventional radiographs, as was also seen in our study (11).

The presence of an intraarticular fracture worsens the prognosis, and MDCT with multiplanar reconstructions is a recommended complementary examination to reveal a possible occult intraarticular fracture of the talus if subtalar joint dislocation or subluxation is suspected. (12)

Talar, calcaneal, tibial, and malleolar fractures should be evaluated for PTT entrapment, since all entrapments associated with one of these fractures involved the PTT. From our findings, the posterior tibialis has the highest likelihood of becoming entrapped in ankle fractures and should be considered with a high index of suspicion in instances of leg tendon entrapment. Both tendon dislocation and entrapment occurred together only in pilon fractures, and these fractures are the most common ankle fractures associated with tendon entrapment. Identification of tendon entrapment on MDCT by the radiologist is important for operative planning. Preexisting knowledge of entrapped structures can influence operative approach (may require additional or alternative incisions) and sequence of dissection and reduction (13).

Calcaneal fractures are the most common fractures of the foot and present a large socioeconomic burden as they are estimated to account for $2 \%$ of all fractures presenting to emergency departments (14).

Calcaneal fractures are often the result of traumatic axial loading from a fall or automobile accident. In the present study, the majority of peroneal tendon dislocations resulted from calcaneal fractures. The Sanders classification to evaluate calcaneal fractures is based on the number of intraarticular fracture lines and their location on CT images (10).

Ranging from type I to type IV, higher classifications are meant to correspond to greater severity, which may have prognostic indications. Sanders type I fractures includes 
intraarticular fractures that have less than $2 \mathrm{~mm}$ of articular displacement, regardless of the number of fracture lines/fragments present and can often be managed nonoperatively (15)

Sanders type II and III fractures have one and two primary fracture line(s), respectively, whereas Sanders type IV involves three or more primary fracture lines with greater than $2 \mathrm{~mm}$ of articular displacement, and are therefore severely comminuted. (15)

Ketz et al. reported a series that corroborated peroneal dislocation on CT with intraoperative findings and found a higher incidence of suspected peroneal subluxation or dislocation on preoperative CT compared to intraoperative findings. In 47 patients with CT evidence of peroneal displacements, there were 36 false positives based on intraoperative exam. (15)

Toussaint et al. reported a three-institution retrospective review querying calcaneal fractures with associated peroneal tendon displacement (displacement was defined as subluxation or dislocation in their series). In 421 calcaneal fractures, peroneal tendon displacement was present in $28 \%$ of cases (118/421). (16)
Importantly, the radiology report only identified peroneal subluxation/dislocation in $10.2 \%$ of cases in their study. They also classified calcaneal fractures according to Essex-Lopresti and Sanders classifications and found significantly higher incidence of peroneal tendon displacement with joint depression compared to tongue-type fractures classified by the former and significantly high rates of displacement with increasing Sanders classification (16).

Kwaadu et al. reported similar findings in a retrospective series of 90 patients with 97 calcaneal fractures. They found Sanders type IV calcaneal fractures were associated with a significantly higher incidence of peroneal dislocation requiring repair compared with type III or lower (11 of 55 [20\%] compared to 2 of 42 [4.8\%]) (17).

In spite of using plain radiography as a routine investigation for hind-foot injuries, it may be not helpful in detecting and diagnosing in many conditions, but is always done to rule out other conditions. CT scanning is a non-invasive common technique and has gained use in identifying sites associated with pain and other pathologies like Achilles Tendonitis, Bursitis, and Plantar Fasciitis and offers more accurate images for this complex 
articulation and facilitate diagnosis especially by coronal axis and 3D images. (18).

So, CT is a commonly used imaging technique after radiography in the setting of ankle and hind-foot fracture. Compared with conventional helical CT, MDCT is faster and has fewer motion artifacts. It offers reduced partial volume effects, decreased image noise, high-quality multiplanar reconstructions, and isotropic viewing, all of which increase its diagnostic power and benefit patients who present to the emergency department with trauma. The position of the ankle or foot is not crucial because the reformats are of excellent quality. The high-quality multiplanar reconstruction capability is especially useful in analyzing complex ankle and foot fractures. (19)

\section{Conclusion}

In complex intra-articular distal tibial, calcaneal and talar fractures, MDCT demonstrates well the degree of articular surface depression, fragment size and position. Data obtained by from volumerendered imaging may assist the clinician in planning the surgical approach. Computed tomography is valuable in the diagnosis of cases of occult syndesmotic injury, especially when the patient cannot tolerate stress radiographs.

\section{References}

1. Haapamaki V V, Kiuru MJ, Koskinen SK. Ankle and foot injuries: analysis of MDCT findings. Am J Roentgenol. 2004;183(3):615-22.

2. Udayasankar UK, Momin Z, Small WC. 3-D Post-processing: Principles and Practical Applications. In: MDCT. Springer; 2008. p. 6579.

3. Peschman JR, Brasel K. Medical Imaging and Injury Scaling in Trauma Biomechanics. In: Accidental Injury. Springer; 2015. p. 51-82.

4. Sebastian S, Salamipour H. Role of MDCT in the evaluation of musculoskeletal trauma. In: MDCT. Springer; 2008. p. 324-32.

5. Geijer M, El-Khoury GY. MDCT in the evaluation of skeletal trauma: principles, protocols, and clinical applications. Emerg Radiol. 2006;13(1):7-18.

6. Rieger M, Czermak B, El Attal R, Sumann G, Jaschke W, Freund M. Initial clinical experience with a 64-MDCT whole-body scanner in an emergency department: better time management and diagnostic quality? J Trauma Acute Care Surg. 2009;66(3):648-57.

7. Caracchini G, Pietragalla M, De Renzis A, Galluzzo M, Carbone M, Zappia M, et al. Talar fractures: radiological and $\mathrm{CT}$ evaluation and classification systems. Acta Bio Medica Atenei Parm. 2018;89(Suppl 1):151.

8. Rogers LF. Radiology of skeletal trauma. 1982; 
9. Atkins R. Pathology of calcaneal fractures. J Bone Jt Surgery-british Vol. 2001;83:326-7.

10. Sanders R. Current concepts review-displaced intra-articular fractures of the calcaneus. JBJS. 2000;82(2):225-50.

11. Ebraheim NA, Biyani A, Padanilam T, Paley K. A pitfall of coronal computed tomographic imaging in evaluation of calcaneal fractures. Foot ankle Int. 1996;17(8):503-5.

12. Bohay DR, Manoli A. Occult fractures following subtalar joint injuries. Foot ankle Int. 1996;17(3):164-9.

13. Eastman JG, Firoozabadi R, Benirschke SK, Barei DP, Dunbar RP. Entrapped posteromedial structures in pilon fractures. J Orthop Trauma. 2014;28(9):528-33.

14. Squires B, Allen PE, Livingstone J, Atkins RM. Fractures of the tuberosity of the calcaneus. J Bone Joint Surg Br. 2001;83(1):55-61.

15. Ketz JP, Maceroli M, Shields E, Sanders RW. Peroneal tendon instability in intra-articular calcaneus fractures: a retrospective comparative study and a new surgical technique. J Orthop Trauma. 2016;30(3):e82-7.
16. Toussaint RJ, Lin D, Ehrlichman LK, Ellington JK, Strasser N, Kwon JY. Peroneal tendon displacement accompanying intra-articular calcaneal fractures. JBJS. 2014;96(4):310-5.

17. Kwaadu KY, Fleming JJ, Florek D. Superior peroneal retinacular injuries in calcaneal fractures. J Foot Ankle Surg. 2015;54(3):458-63.

18. Mohan HK, Gnanasegaran G, Vijayanathan S, Fogelman I. SPECT/CT in imaging foot and ankle pathology - the demise of other coregistration techniques. In: Seminars in nuclear medicine. Elsevier; 2010. p. 41-51.

19. Rydberg J, Buckwalter KA, Caldemeyer KS, Phillips MD, Conces Jr DJ, Aisen AM, et al. Multisection CT: scanning techniques and clinical applications. Radiographics. 2000;20(6):1787-806.

To cite this article: Medhat M. Refaat, Ahmed E. Shalaan, Esraa F. Abdl-Khalek. Role of Multislice CT in Evaluation of Traumatic Hind-Foot Injuries. BMFJ 2022; 39(Radiology):220-233. DOI:10.21608/bmfj.2021.88788.1449 